\title{
ANALYTIC AND DIFFERENTIABLE FUNCTIONS VANISHING ON AN ALGEBRAIC SET
}

\author{
WOJCIECH KUCHARZ
}

(Communicated by Irwin Kra)

\begin{abstract}
Let $U$ be an open semi-algebraic subset of $\mathbf{R}^{n}$ and let $X$ be a closed analytic subset of $U$ which is also a semi-algebraic set (e.g., $U=\mathbf{R}^{n}$ and $X$ is an algebraic subset of $\mathbf{R}^{n}$ ). It is proved that the ideal of analytic functions on $U$ vanishing on $X$ is finitely generated provided that the set $X$ is coherent. The ideal of infinitely differentiable functions on $U$ vanishing on $X$ is finitely generated if and only if the set $X$ is coherent.
\end{abstract}

1. The results. Let $U$ be an open subset of $\mathbf{R}^{n}$. Denote by $O(U)$ and $\mathcal{E}(U)$ the ring of analytic functions and $C^{\infty}$ functions on $U$, respectively. Given a subset $X$ of $U$, we define $I(X)$ to be the ideal of $O(U)$ and $I_{*}(X)$ to be the ideal of $\mathcal{E}(U)$ of all functions vanishing on $X$. It would be interesting to know under what assumptions on $X$ the ideals $I(X)$ and $I_{*}(X)$ are finitely generated (cf. $\left.[1,4]\right)$. For instance, it remains an open question whether the ideal $I(X)$ is finitely generated if $X$ is an algebraic subset of $\mathbf{R}^{n}[\mathbf{4}$, p. 65].

We propose one result in this direction. Let $O$ be the sheaf of germs of analytic functions on $\mathbf{R}^{n}$ and let $J(X)$ be the subsheaf of ideals of $O \mid U$ of germs vanishing on $X$. Recall that if $X$ is a closed analytic subset of $U$, then $X$ is said to be coherent if the sheaf $J(X)$ is coherent. Denote by $\mathcal{E}$ the sheaf of germs of $C^{\infty}$ functions on $\mathbf{R}^{n}$ and by $J_{*}(X)$ the subsheaf of ideals of $\mathcal{E} \mid U$ of germs vanishing on $X$. We shall need the following characterization of coherent analytic sets.

PROPOSITION 1. Let $U$ be an open subset of $\mathbf{R}^{n}$ and let $X$ be a closed analytic subset of $U$. Then $X$ is coherent if and only if for each $x$ in $U$ the stalk $J_{*}(X)_{x}$ is finitely generated over $\mathcal{E}_{x}$.

Let us recall that a subset of $\mathbf{R}^{n}$ is said to be semi-algebraic if it belongs to the smallest family of subsets which contains sets of the form $\left\{x \in \mathbf{R}^{n} \mid p(x)>0\right\}$, where $p: \mathbf{R}^{n} \rightarrow \mathbf{R}$ is a polynomial function, and which is closed under the Boolean operations of finite union, finite intersection and taking complements. Obviously, every algebraic subset of $\mathbf{R}^{n}$ is semi-algebraic.

THEOREM 2. Let $U$ be an open semi-algebraic subset of $\mathbf{R}^{n}$ and let $X$ be a closed analytic subset of $U$ which is also a semi-algebraic set. Then:

(i) The ideal $I(X)$ is finitely generated if the set $X$ is coherent.

(ii) The ideal $I_{*}(X)$ is finitely generated if and only if the set $X$ is coherent.

REMARK 3. (i) If $X$ is a closed coherent analytic subset of $\mathbf{R}^{n}$, then, in general, the ideal $I(X)$ is not finitely generated (cf. [3] for an idea of how to construct an example).

Received by the editors March 5, 1986.

1980 Mathematics Subject Classification (1985 Revision). Primary 32K15, 32B15. 
(ii) Theorem 2 applies, in particular, in the case where $U=\mathbf{R}^{n}$ and $X$ is an algebraic subset of $\mathbf{R}^{n}$. We wish to point out that in such a case the ideal $I(X)$ is not, in general, generated by polynomials. For example, the curve $X$ in $\mathbf{R}^{2}$ given by the equation $x_{2}^{2}-x_{1}^{2}\left(x_{1}-1\right)=0$ is irreducible as an algebraic set and has an isolated point at the origin. It follows that the ideal $I(X)$ cannot be generated by polynomials. Clearly, $X$ is coherent as an analytic set.

There is a conjecture (cf. $[4$, p. 65]) that the ideal $I(X)$ is always generated by so-called Nash functions if $X$ is an algebraic subset of $\mathbf{R}^{n}$.

Let us mention that if $Y$ is a complex algebraic subset of $\mathbf{C}^{n}$, then the ideal of holomorphic functions on $\mathbf{C}^{n}$ vanishing on $Y$ is generated by polynomials and, hence, is finitely generated [9].

PROPOSITION 4. Let $X$ be an algebraic subset of $\mathbf{R}^{n}$. If the set of singular points of $X$ is bounded, then the ideal $I(X)$ is finitely generated.

We conclude this section by giving

EXAMPLE 5. Let $f\left(x_{1}, x_{2}, x_{3}\right)=x_{3}\left(x_{1}^{2}+x_{2}^{2}\right)-x_{1}^{3}$ and let $X$ be the set of zeros of $f$. One checks easily that the set of singular points of $X$ is not bounded and $X$ is not coherent as an analytic set. However, the ideal $I(X)$ is generated by $f[2$, Proposition 5.2(1)]. By Theorem 2, the ideal $I_{*}(X)$ is not finitely generated. (It is stated in [2, p. 85] that the ideal $I_{*}(X)$ is not generated by $f$ but the proof of this fact is incorrect.)

2. The proofs. The proofs are based on the results of Malgrange, Merrien and Tougeron.

ProOF OF Proposition 1. If the set $X$ is coherent, then $J_{*}(X)=J(X) \mathcal{E} \mid U$ $[12$, p. 127, Theorem 4.2]. Since each stalk of $J(X)$ is finitely generated so is each stalk of $J_{*}(X)$.

Now assume that each stalk of $J_{*}(X)$ is finitely generated. Let $\mathcal{F}_{n}$ be the $\mathbf{R}$ algebra of formal power series in $n$ variables. Given a point $x$ in $\mathbf{R}^{n}$, denote by $T_{x}: \mathcal{E}_{x} \rightarrow \mathcal{F}_{n}$ the homomorphism induced by the infinite Taylor expansion at $x$. It follows from [7, p. 90, Theorem 3.5] that $T_{x}\left(J_{*}(X)_{x}\right)=T_{x}\left(J(X)_{x}\right) \mathcal{F}_{n}$ for all $x$ in $U$. Fix a point $x$ in $U$ and let $f_{1}, \ldots, f_{k}$ be analytic functions defined on a neighborhood $U_{x}$ of $x$ in $U$ whose germs $f_{1 x}, \cdots, f_{k x}$ at $x$ generate $J(X)_{x}$. Then $T_{x}\left(f_{1}\right), \ldots, T_{x}\left(f_{k}\right)$ generate $T_{x}\left(J_{*}(X)_{x}\right)$. Clearly, $J_{*}(X)_{x}$ is a closed ideal of $\mathcal{E}_{x}$ (cf. [12, pp. 98-99] for a definition of a closed ideal in $\mathcal{E}_{x}$ ). By [5, p. 48, Proposition 1], the germs $f_{1 x}, \ldots, f_{k x}$ generate $J_{*}(X)_{x}$. Since, by [1, Proposition 1.3], the sheaf $J_{*}(X)$ is quasi-flasque, the germs $f_{1 y}, \cdots, f_{k y}$ generate $J_{*}(X)_{y}$ for all $y$ in $U_{x}$ provided that $U_{x}$ is a sufficiently small neighborhood of $x[12$, p. 115, Proposition 6.4]. Therefore $T_{y}\left(f_{1}\right), \ldots, T_{y}\left(f_{k}\right)$ generate $T_{y}\left(J(X)_{y}\right) \mathcal{F}_{n}$. By [12, p. 26, Proposition 8.2], the germs $f_{1 y}, \ldots, f_{k y}$ generate $J(X)_{y}$ for all $y$ in $U_{x}$ and, hence, $X$ is a coherent set.

PROOF OF THEOREM 2. We claim that there exists a positive integer $k$ such that the stalk $J(X)_{x}$ can be generated by at most $k$ elements of $O_{x}$ for all $x$ in $U$. Indeed, define $h: \mathbf{R}^{n} \rightarrow \mathbf{R}^{n}$ by $h(x)=x /\left(1+\|x\|^{2}\right)^{1 / 2}$ for $x$ in $\mathbf{R}^{n}$. Since the graph of $h$ is semi-algebraic, the set $Y=h(X)$ is also semi-algebraic [10]. By 
[8, Theorem 7.1], the subsheaf $J(Y)$ of $O$ over $\mathbf{R}^{n}$ is semifinite. Thus for each point $x$ in $\mathbf{R}^{n}$ there exist a neighborhood $U_{x}$ of $x$ in $\mathbf{R}^{n}$ and a positive integer $k_{x}$ such that $J(Y)_{y}$ can be generated by at most $k_{x}$ elements of $O_{y}$ for all $y$ in $U_{x}$. Since $h$ diffeomorphically maps $\mathbf{R}^{n}$ onto the open unit ball in $\mathrm{R}^{n}$, the set $Y$ is bounded and the claim follows.

If $X$ is coherent, then the ideal $\Gamma(U, J(X))$ of global sections of $J(X)$ is finitely generated (cf. [6]). Since, by Theorem A of Cartan, $I(X)=\Gamma(U, J(X))$, the proof of (i) is completed.

Again, if $X$ is coherent, then $J_{*}(X)=J(X) \mathcal{E} \mid U[\mathbf{1 2}$, p. 127, Theorem 4.2]. It follows from [12, p. 214, Proposition 5.1] that the ideal $I_{*}(X)=\Gamma\left(U, J_{*}(X)\right)$ is finitely generated.

Now assume that the ideal $I_{*}(X)$ is finitely generated. Clearly, $J_{*}(X)_{x}=$ $I_{*}(X) \mathcal{E}_{x}$ for all $x$ in $U$. By Proposition 1 , the set $X$ is coherent.

PrOOF OF PrOPOSITION 3. Let $J$ be the sheaf of ideals associated with $I(X)$, i.e., $J_{x}=I(X) O_{x}$ for $x$ in $\mathbf{R}^{n}$. By [11, Theorem 2], the sheaf $J$ is coherent. Note that if $x$ is a nonsingular point in $x$, then the ideal $J_{x}$ is generated by polynomials vanishing on $X$. Since the set of singular points of $X$ is bounded, there exists a positive integer $k$ such that the ideal $J_{x}$ can be generated by at most $k$ elements of $O_{x}$ for all $x$ in $\mathbf{R}^{n}$. It follows that the ideal $I(X)=\Gamma(X, J)$ is finitely generated.

\section{BIBLIOGRAPHY}

1. W. A. Adkins and J. V. Leahy, Criteria for finite generation of ideals of differentiable functions, Duke Math. J. 42 (1975), 707-716.

2. _ـ A global real analytic nullstellensatz, Duke Math. J. 43 (1976), 81-86.

3. J. Becker, Parametrizations of analytic varieties, Trans. Amer. Math. Soc. 183 (1973), 265292.

4. J. Bochnak and J. J. Risler, Analyse différentiale et géométrie analytique, quelques questions ouvertes, singularités d'applications différentiables, Lecture Notes in Math., vol. 535, Springer, 1976, pp. 63-69.

5. __ Sur la divisibilité des fonctions différentiables, singularités d'applications différentiables Lecture Notes in Math., vol. 535, Springer, 1976, pp. 45-62.

6. O. Forster, Zur Theorie der Steinichen Algebren und Moduln, Math. Z. 97 (1967), 376-405.

7. B. Malgrange, Ideals of differentiable functions, Oxford Univ. Press, 1966.

8. J. Merrien, Faisceaux analytiques semi-cohérents, Ann. Inst. Fourier (Grenoble) 30 (1980), 165-219.

9. W. Rudin, A geometric criterion for algebraic varieties, J. Math. Mech. 17 (1968), 671-683.

10. A. Seidenberg, A new decision method for elementary algebra, Ann. of Math. (2) 60 (1954), 365-374.

11. Y. T. Siu, Noetherianness of rings of holomorphic functions on Stein compact subsets, Proc. Amer. Math. Soc. 21 (1969), 483-489.

12. J. Cl. Tougeron, Idéaux de fonctions différentiables, Ergebnisse der Math. 71, Springer-Verlag, 1972.

Department of Mathematics and Statistics, University of New Mexico, Albuquerque, NeW Mexico 87131 